\title{
Subepithelial myofibroblast in lung adenocarcinoma: a histological indicator of excellent prognosis
}

\author{
Daisuke Matsubara ${ }^{1}$, Teppei Morikawa ${ }^{2}$, Akiteru Goto ${ }^{2}$, Jun Nakajima ${ }^{3}$, Masashi Fukayama ${ }^{2}$ \\ and Toshiro Niki ${ }^{1,2}$ \\ ${ }^{1}$ Department of Pathology, Jichi Medical University, Shimotsuke, Japan; ${ }^{2}$ Department of Pathology, Graduate \\ School of Medicine, the University of Tokyo, Bunkyo-ku, Japan and ${ }^{3}$ Department of Cardiothoracic Surgery, \\ the University of Tokyo, Bunkyo-ku, Japan
}

\begin{abstract}
We report here the presence of subepithelial myofibroblasts in pure bronchioloalveolar carcinoma and a subset of invasive lung adenocarcinoma. The subepithelial myofibroblasts we describe were observed in a peculiar location beneath the cancer cells in the alveolar septa. Immunohistochemically, they were positive for $\alpha$-smooth muscle actin and calponin, but negative for desmin and h-caldesmon. To gain insight into their biological significance, we examined 116 surgically resected lung adenocarcinomas. The resected tumors included 13 bronchioloalveolar carcinomas, 20 mixed type adenocarcinomas with bronchioloalveolar carcinoma components, 57 papillary adenocarcinomas, 22 solid adenocarcinomas with mucin, and 4 acinar adenocarcinomas. All specimens were immunostained for $\alpha$-smooth muscle actin to visualize the myofibroblasts. In all of the pure bronchioloalveolar carcinomas observed, the subepithelial myofibroblasts were completely preserved adjacent to the cancer cells. In mixed adenocarcinomas with bronchioloalveolar carcinoma components, subepithelial myofibroblasts were present in the bronchioloalveolar carcinoma components, but scanty in the invasive areas, where stromal myofibroblasts emerged between the cancer cell nests. Subepithelial myofibroblasts were retained, however, in the invasive areas of a subset of invasive adenocarcinomas. Survival analysis showed that the retention of subepithelial myofibroblasts in these invasive tumors was associated with low rates of lymphatic and vascular invasion, a low rate of lymph node involvement, and an excellent patient survival. These results suggest that subepithelial myofibroblasts increase in bronchioloalveolar carcinomas, but are gradually replaced by typical stromal myofibroblasts during progression into invasive cancer. A subset of invasive adenocarcinomas retains subepithelial myofibroblasts. Analysis of subepithelial myofibroblasts may be helpful in identifying a subset of lung adenocarcinoma with excellent prognosis.

Modern Pathology (2009) 22, 776-785; doi:10.1038/modpathol.2009.27; published online 27 March 2009
\end{abstract}

Keywords: lung cancer; prognosis; myofibroblast; immunohistochemistry

Lung cancer is the leading cause of cancer mortality in USA, Japan, and other developed countries. ${ }^{1}$ Moreover, the incidence of lung adenocarcinoma is widely recognized to be increasing among industrialized countries. ${ }^{2}$ The prognosis of lung carcinoma is poor: even if diagnosed successfully, patients with stage I lung carcinoma have a 5-year survival rate of only $70 \%$ after surgical resection. ${ }^{3}$

In 1995, Noguchi et $a l^{4}$ proposed a new histological classification for lung adenocarcinomas of small

Correspondence: Dr T Niki, MD, Department of Pathology, Jichi Medical University, 3311-1 Yakushiji, Shimotsuke 329-0498, Tochigi, Japan.

E-mail: tniki@jichi.ac.jp

Received 1 August 2008; revised 5 December 2008; accepted 8 December 2008; published online 27 March 2009 size (maximum dimension $2 \mathrm{~cm}$ or less). In survival analysis, tumors of $<2 \mathrm{~cm}$ in diameter with a pure lepidic growth pattern (Noguchi type A) and tumors showing bland, elastotic sclerosis without active fibroblastic proliferation (Noguchi type B) had excellent prognosis with no lymph node metastasis. In contrast, tumors with 'active fibroblasts' (Noguchi type C) had a poorer outcome. ${ }^{4}$ These results suggest that characteristic stromal alterations accompany the invasion of cancer cells, and that stromal myofibroblasts or cancer-associated fibroblasts $(\mathrm{CAFs}),{ }^{5}$ that is, 'active fibroblasts' ${ }^{4}$ are crucial in this process. Stromal myofibroblasts are also reported to facilitate invasion and metastasis of cancer cells through cancer-stromal cell interaction. ${ }^{6}$

Lung cancer develops and progresses through an accumulation of gene abnormalities in oncogenes 
and tumor suppressor genes. ${ }^{7}$ However, the stromal changes that occur during progression from pure bronchioloalveolar carcinoma (type A or B of Noguchi's classification) to mixed type adenocarcinoma with a bronchioloalveolar carcinoma component (type C of Noguchi's classification) ${ }^{8}$ and to pure invasive adenocarcinoma (types $\mathrm{E}, \mathrm{D}$, and $\mathrm{F}$ of Noguchi's classification) are not well documented in the literature.

In this report we documented the presence of peculiar myofibroblasts, what we call 'subepithelial myofibroblasts,' in pure bronchioloalveolar carcinoma, and characterized the immunohistochemical profile of these cells on the basis of their expression of $\alpha$-smooth muscle actin, calponin, desmin, and hcaldesmon. Next, in an evaluation of the presence of subepithelial myofibroblasts in invasive lung adenocarcinomas, we found that these cells were lost in most invasive adenocarcinomas, but were preserved in a subset of invasive adenocarcinomas. Finally, we showed that the retention of subepithelial myofibroblasts in patients with invasive lung adenocarcinomas was associated with low rates of lymphatic and vascular invasion, a low rate of lymph node involvement, and an excellent patient survival. To our knowledge, this is the first report to document the presence and prognostic significance of subepithelial myofibroblasts in lung adenocarcinomas.

\section{Materials and methods}

\section{Patients and Tumors}

Tumor specimens were obtained from 116 patients who underwent lung cancer surgery at the University of Tokyo hospital during the period from August 1999 to April 2003. The patients included 77 males and 39 females, ranging in age from 38 to 90 years (average 65 years). The observation period ranged from 0.8 to 57.3 months, with a median follow-up period of 31.4 months. The cases were staged according to the tumor-node-metastasis system adopted by the American Joint Committee on Cancer and the International Union against Cancer. ${ }^{9}$ Eighty-two were stage I (54 stage IA and 28 stage IB), 13 were stage II (four stage IIA and nine stage IIB), and 21 were stage III (13 stage IIIA and 8 stage IIIB). No stage IV cases were found. The adenocarcinomas were histologically subtyped according to the World Health Organization classification for tumors. ${ }^{10}$ As most lung adenocarcinomas consist of heterogeneous histological components, dominant subtypes were mainly documented. ${ }^{11,12}$ Mixed type tumors mainly consisting of a bronchioloalveolar carcinoma component were classified as mixed subtype adenocarcinomas with bronchioloalveolar carcinoma components. On the basis of this approach, the histological typing of these tumors revealed 13 pure bronchioloalveolar carcinomas, 57 papillary adenocarcinomas, 4 acinar adenocarcinomas, 22 solid adenocarcinomas with mucin, and 20 mixed subtype adenocarcinomas with bronchioloalveolar carcinoma components. The histological criteria for vascular, lymphatic, and pleural invasion were as described earlier. ${ }^{13,14}$ Stromal invasion was evaluated according to the criteria described by Terasaki et al. ${ }^{8}$

\section{Immunohistochemistry}

The specimens for immunohistochemical evaluation were taken from representative areas of the tumor, including the largest cut surface. When it was felt that this was not sufficient owing to intratumor heterogeneity, additional cut surface of tumors were further taken as appropriate. Thus, a total of 126 sections were submitted to immunohistochemical analysis.

Five-micrometer-thick sections were cut and deparaffinized through graded xyline and ethanol. The sections were first treated with $0.3 \%$ hydrogen peroxide in methanol for 30 min to block endogenous peroxidase activity. Then sections were either autoclaved in $10 \mathrm{mM}$ citrate buffer $(\mathrm{pH}$ 6.0) for $10 \mathrm{~min}$ at $120^{\circ} \mathrm{C}$ (for $\alpha$-smooth muscle actin, calponin, h-caldesmon, and desmin). Sections were then preincubated with $10 \%$ normal horse serum in phosphate-buffered saline, incubated with a mouse monoclonal antibody against $\alpha$-smooth muscle actin (Sigma, clone 1A4) at a dilution of 1:200, mouse monoclonal antibody against calponin (Dako, M3556) at 1:200, mouse monoclonal antibody against caldesmon (Dako, M3557) at 1:100 at $4^{\circ} \mathrm{C}$ overnight, and then with biotinylated secondary antibody for $45 \mathrm{~min}$. Subsequently, the sections were allowed to react for $30 \mathrm{~min}$ with avidin-biotinylperoxidase complex (ABC) using a Vectastain ABC kit (Vector Laboratories) and subjected to the peroxidase reaction using $0.02 \% 3,3^{\prime}$-diaminobenzidine tetrahydrochloride (DAB) as a chromogen in Tris-HCl buffer (pH 7.6) containing $0.007 \%$ hydrogen peroxide. No significant staining was observed in the negative controls, which were prepared using the same class of mouse immunoglobulin at the same concentration.

\section{Evaluation of Subepithelial Myofibroblasts}

We classified tumor areas into four patterns according to the degree of subepithelial myofibroblast retention around tumor cells: pattern A, tumor cells are completely surrounded by a regular and thin layer of subepithelial myofibroblasts; pattern B, almost all of the tumor cells are surrounded by irregular layers of subepithelial myofibroblasts, with focal disruption of layers $(<5 \%)$ in some areas; pattern C, subepithelial myofibroblasts are significantly $(<50 \%)$ lost around the tumor cells; pattern $\mathrm{D}, \geq 50 \%$ of subepithelial myofibroblasts are lost around the tumor cells. 
We then divided the lung adenocarcinomas into three groups on the basis of the dominant pattern and the presence or absence of foci showing pattern C or pattern D: group I, tumors predominantly showing pattern $A$ or pattern $B$ without foci showing $>5-\mathrm{mm}$ pattern $\mathrm{C}$ or $\mathrm{D}$; group II, tumors with foci showing $>5-\mathrm{mm}$ pattern $\mathrm{C}$ and without foci showing $>5-\mathrm{mm}$ pattern $\mathrm{D}$; group III, tumors with foci showing $>5-\mathrm{mm}$ pattern $\mathrm{D}$. We adopted a cutoff value of $5 \mathrm{~mm}$ because this cutoff value has often been used in earlier reports. ${ }^{8,15}$ The pattern of subepithelial myofibroblasts was evaluated by two pathologists (TN and DM). The concordance rate was $87 \%$, and the discrepancies were resolved by a joint session.

\section{Statistics}

The $\chi^{2}$-test was used to evaluate the clinicopathological correlations. Survival curves were generated using the Kaplan-Meier method and differences in survival were analyzed by the log-rank test. The log-rank test was used to identify the univariate predictors. Variables, which showed significant difference in survival in univariate analysis, were included in a multivariate Cox model. The results were considered significant if the $P$-value was $<0.05$. All statistical calculations were performed using the StatView computer program (Abacus Concepts, Berkeley, USA).

\section{Results}

\section{Characteristics of Subepithelial Myofibroblasts}

It has been reported that alveolar interstitium in the normal lung contains myofibroblasts that stain positive for vimentin, but negative for $\alpha$-smooth muscle actin. ${ }^{16-18}$ In keeping with these earlier reports, $\alpha$-smooth muscle actin-positive cells were only rarely seen within the normal alveolar septa in our analysis. $\alpha$-smooth muscle actin staining of small sized pure bronchioloalveolar carcinoma is shown in Figure 1. In contrast to normal lung, $\alpha$-smooth muscle actin-positive cells were present in the area of bronchioloalveolar carcinoma and at higher magnification, $\alpha$-smooth muscle actin-positive myofibroblasts appeared abruptly at the interface between the normal lung and bronchioloalveolar carcinoma (Figure 1a and b). Close examination of these $\alpha$-smooth muscle actin-positive cells showed that these cells were slender in shape and localized immediately beneath the cancer cells (Figure 1c and d). Owing to their slender profile, their nuclei were only occasionally observable. Their $\alpha$-smooth muscle actin-positive cytoplasm, on the other hand, could be easily recognized as a thin linear extension along the alveolar septa (Figure 1c) or surrounding neoplastic acinar structure, especially when the alveolar septa were thickened by fibrosis (Figure 2a).

To confirm the myofibroblastic nature of subepithelial fibroblast, we performed immunohistochemical analysis using additional myogenic markers. Subepithelial myofibroblasts were positive for calponin (Figure 2b), but were negative for desmin (Figure 2c) and h-caldesmon (Figure 2d), consistent with their myofibroblastic nature.

Unable to recognize the precise location of these cells by light microscopy, we examined the cells by transmission electron microscopy. As shown in Figure 3a and b, subepithelial myofibroblasts were located underneath the basal lamina and embedded in collagenous matrix. At high magnification, rough endoplasmic reticulum was abundantly seen in the cytoplasm. Dense bodies were also sparsely observed, confirming again their myofibroblastic nature (Figure $3 \mathrm{~b}$ ).

\section{Myofibroblasts in Invasive Lung Adenocarcinoma}

Next, we investigated whether subepithelial myofibroblasts were present in invasive lung adenocarcinomas. In mixed type adenocarcinoma with bronchioloalveolar carcinoma components, subepithelial myofibroblasts were retained in the peripheral areas with lepidic growth patterns, as in pure bronchioloalveolar carcinoma. But the myofibroblastic layer that surrounded the neoplastic acinar structure became discontinuous in areas of central collapse and fibrosis (Figure 4a). The subepithelial myofibroblasts also showed a gradual pattern of irregular proliferation obscuring the sharp contour of the myofibroblastic layer (Figure 4b). The subepithelial myofibroblasts were lost in the frankly invasive adenocarcinomas, with bundles of myofibroblasts (stromal myofibroblasts) typically emerging in their place (Figure 4c). In the papillary adenocarcinomas, subepithelial myofibroblasts were faintly visible or completely lost in the preexisting alveolar structure (Figure 4d). Subepithelial myofibroblasts were completely absent in the papillary structure that projected into the alveolar space (Figure 4d). The proliferation of stromal fibroblasts was inconspicuous in some of the papillary adenocarcinomas.

\section{Classification of Lung Adenocarcinomas According to Subepithelial Myofibroblastic Pattern}

To investigate the significance of subepithelial myofibroblasts in lung adenocarcinomas, we classified tumor areas into four patterns according to the degree of subepithelial myofibroblast retention (see Methods). The tumors were then divided into three groups (groups I to III) on the basis of the dominant pattern, using a cutoff value of $5 \mathrm{~mm}$ (see Methods).

Table 1 shows the correlations between histological features and this classification is according to the 

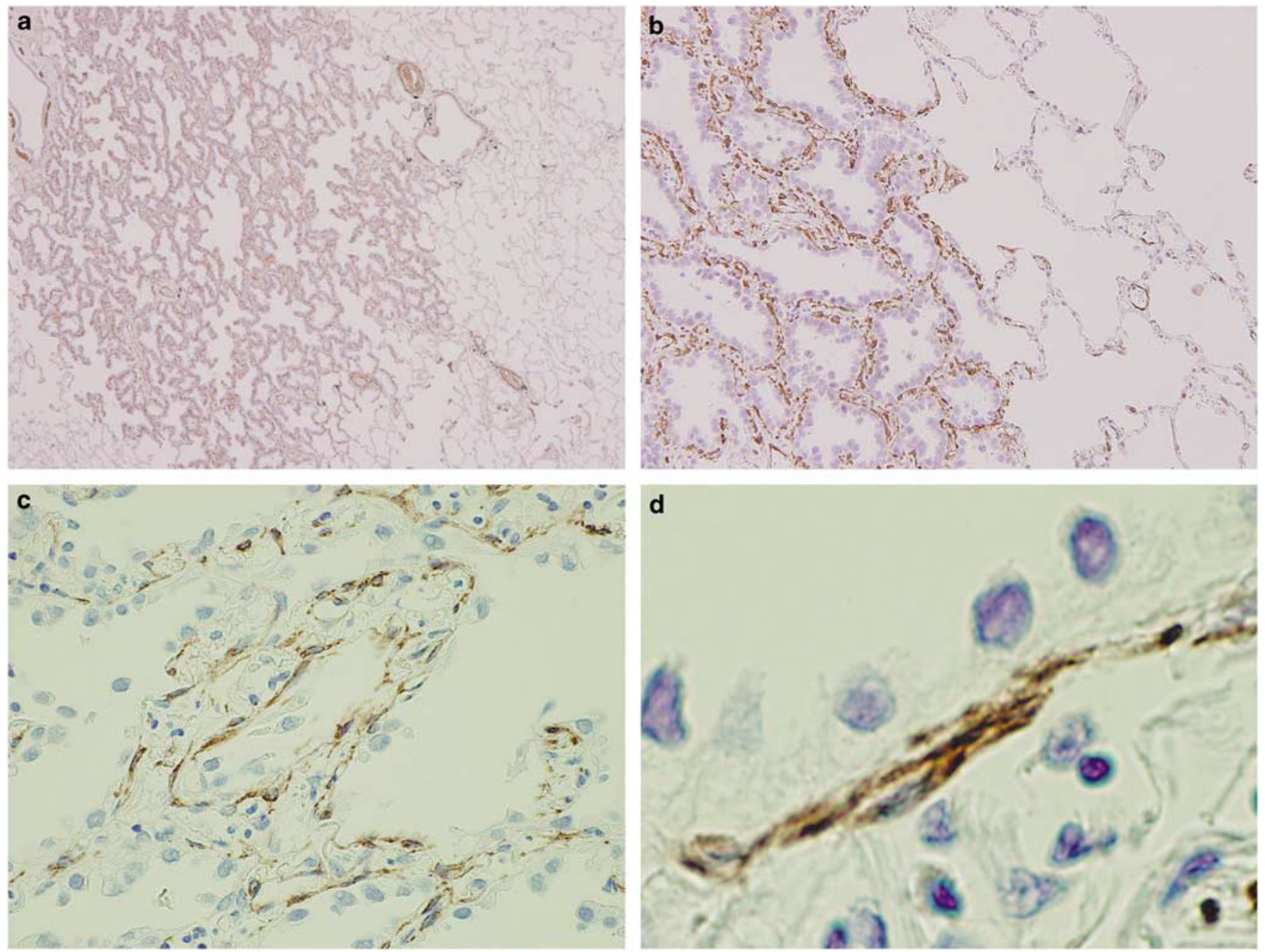

Figure 1 Subepithelial myofibroblasts in pure bronchioloalveolar carcinoma. At lower magnification, $\alpha$-smooth muscle actin-positive cells could be seen in the area of bronchioloalveolar carcinoma, but rarely seen in the adjacent normal lung (a). Subepithelial myofibroblasts positive for $\alpha$-smooth muscle actin abruptly appear at the interface between normal lung and bronchioloalveolar carcinoma. $\alpha$-smooth muscle actin staining in normal alveolar septa represents vascular pericytes (b). In pure bronchioloalveolar carcinoma, a thin layer of slender subepithelial myofibroblasts was observed in the alveolar septa (c). At higher magnification, the cells were located immediately beneath the cancer cells (d).

myofibroblastic pattern. All of the bronchioloalveolar carcinoma cases $(n=13)$ belonged to group I. In contrast, the invasive adenocarcinomas $(n=103)$ consisted of 38 group I tumors, 33 group II tumors, and 32 group III tumors.

\section{Clinicopathological Correlations with Subepithelial Myofibroblastic Pattern}

Patients with bronchioloalveolar carcinoma show an excellent prognosis with $100 \%$ survival. ${ }^{4}$ By definition, bronchioloalveolar carcinomas are free of vascular, lymphatic, and pleural invasion. ${ }^{10}$ To clarify the clinicopathological significance of subepithelial myofibroblasts in invasive lung adenocarcinoma, we evaluated the correlations between the classification (group I to III) according to the myofibroblastic pattern and various clinicopathological parameters in invasive adenocarcinoma $(n=103)$. The results are shown in Table 2 . The classification according to the myofibroblastic pattern was significantly correlated with low rates of lymphatic invasion $(P<0.0001)$, vascular invasion $(P<0.0001)$, pleural invasion $(P=0.0003)$, differentiation $(P<0.0001)$, and lymph node metastasis $(P<0.0001)$, whereas it showed no correlation with pulmonary metastasis $(P=0.5425)$.

\section{Prognostic Significance of the Myofibroblastic Pattern Classification}

The survival curves according to the histological subtyping are shown in Figure 5. bronchioloalveolar carcinoma showed the best prognosis (the 5 -year survival rate $=100 \%$ ), and solid carcinoma with mucin showed the worst prognosis (the 5 -year survival rate $=55 \%$ ). The 5-year survival rates for mixed subtype adenocarcinoma, papillary 

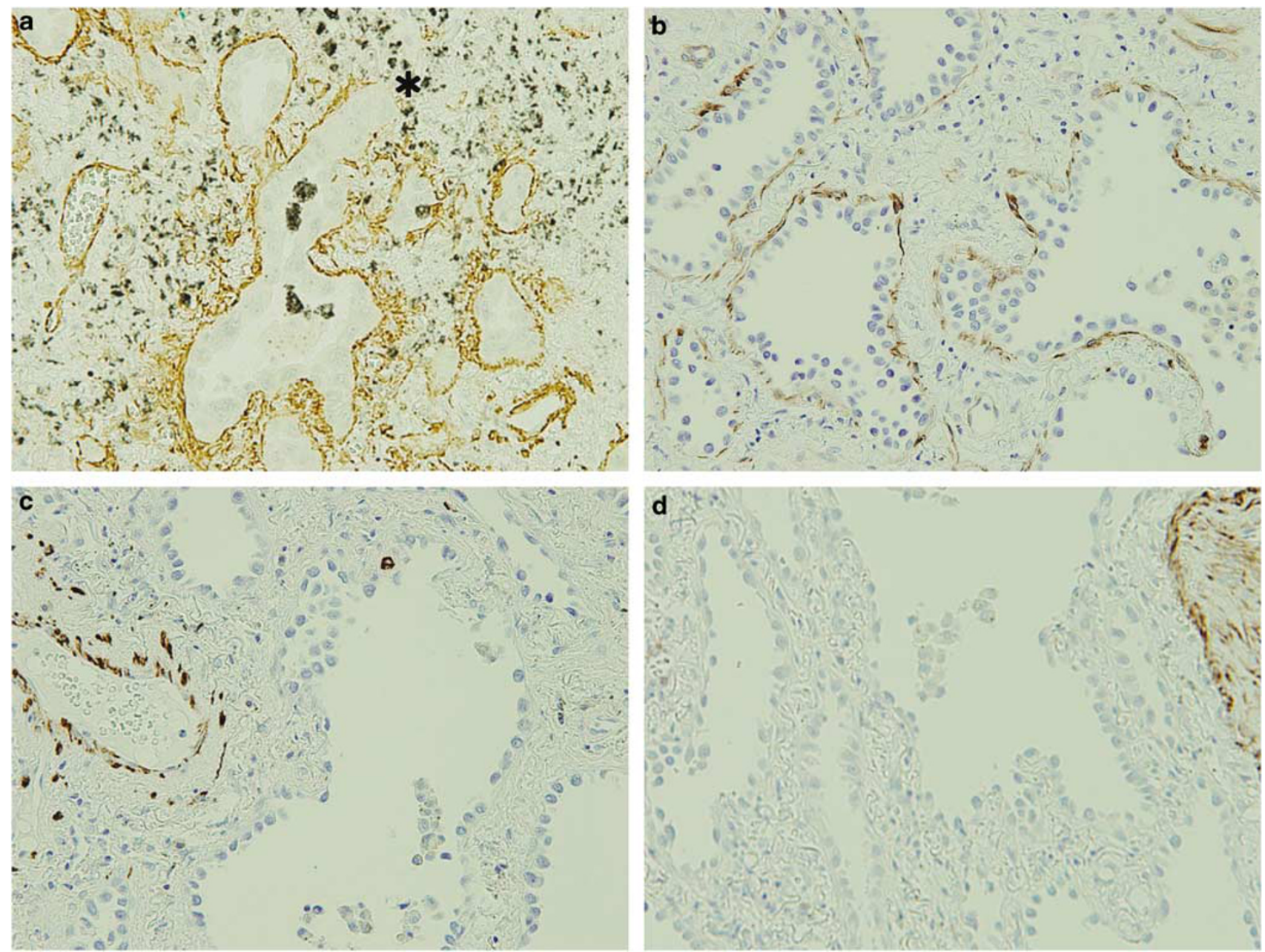

Figure 2 In areas of collapse, the lining of subepithelial myofibroblasts around neoplastic acinar structure was virtually continuous. Focal disruption in the lining of subepithelial myofibroblasts (asterisk) was present in some cases (a). Subepithelial myofibroblasts were positive for calponin (b) but negative for desmin (c) and h-caldesmon (d).

adenocarcinoma, and acinar adenocarcinoma were 85,75 , and $75 \%$, respectively.

The survival curves according to the classification by the myofibroblastic pattern are shown in Figure 6. In this survival analysis, bronchioloalveolar carcinoma cases were excluded because we wanted to ascertain the clinical significance of subepithelial myofibroblasts in the invasive lung adenocarcinoma. As shown in Figure 6a, group I showed a significantly better prognosis than group II and group III, and group III showed the worst prognosis $(P<0.0001$, Mantle-Cox method). The 5-year survival rates for patients with group I, group II, and group III were 97, 66, and $37 \%$, respectively, excluding patients with bronchioloalveolar carcinoma. Close comparison between Figures 5 and 6a clearly showed that in addition to the 13 patients with bronchioloalveolar carcinoma, a subgroup of 38 patients with excellent prognosis, ie, patients with group I tumors, could be separated from the 103 patients with invasive adenocarcinomas when our new histological classification is applied.
Next, invasive adenocarcinoma cases were subdivided into early stage (stage I, $n=69$ ) and advanced stages (stage II and stage III, $n=34$ ) for a survival analysis in each subset. In the early stage (stage I), significant correlations were found between the classification according to the myofibroblastic pattern and survival (the 5-year survival rates for group I $(n=36)$, group II $(n=22)$, and group III $(n=11)$ were 97,81 , and $18 \%$, respectively $(P<0.0001$, Mantle-Cox method)) (Figure 6b). In contrast, no significant correlations were found when advanced-stage tumors were analyzed $(P=0.3269$, Mantle-Cox method).

\section{Multivariate Analysis of Overall Survival Among Pathological Factors}

In the univariate analysis, stage, and various histopathological parameters such as tumor size, lymphatic invasion, vascular invasion, and pleural invasion were statistically correlated with patient 

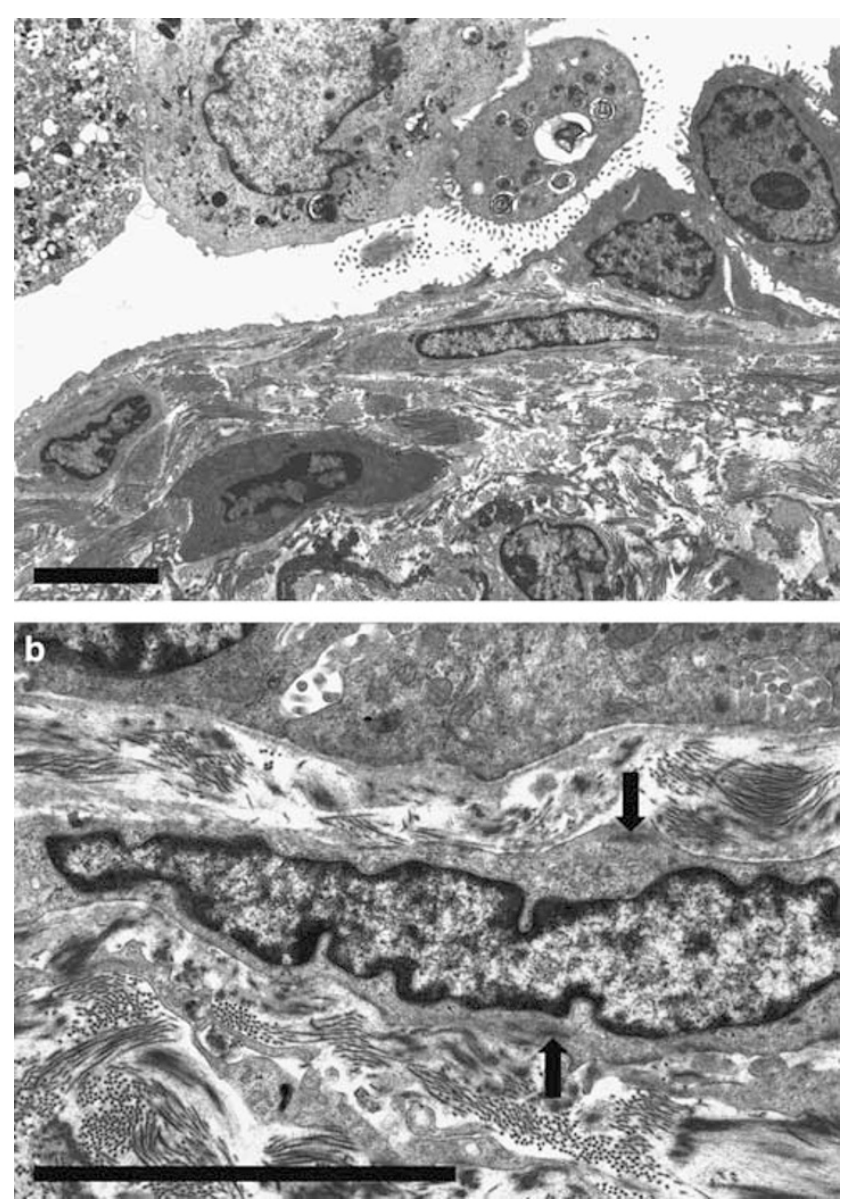

Figure 3 Transmission electron microscopy of subepithelial myofibroblasts. Subepithelial myofibroblasts were located underneath the basal lamina and embedded in collagenous matrix (a). At high magnification, abundant rough endoplasmic reticulum and occasional dense bodies (arrows) were noted (b). Scale $\mathrm{bar}=5 \mu \mathrm{m}$.

prognosis (Table $3 \mathrm{~A})$. In the multivariate analysis stratified for stage, however, only the classification according to the myofibroblastic pattern remained significant (Table 3B).

\section{Discussion}

Pathologists usually rely on architectural distortion and stromal response to recognize invasion in epithelial tumors. Overall, however, the extent of the architectural changes that occur in the myofibroblasts are not well documented for lung adenocarcinoma in the early non-invasive stage or during progression into invasive carcinoma. ${ }^{19,20}$

In this study, we found two types of myofibroblasts in lung adenocarcinoma: stromal myofibroblasts and subepithelial myofibroblasts. The former correspond to so-called cancer-associated myofibroblasts (CAFs), and their emergence is correlated with invasion and poor prognosis. ${ }^{4,5}$ In contrast, the latter have not been described for lung cancer, and the very presence of these cells has remained unrecognized to date. In our analysis, however, the subepithelial myofibroblasts were found in all of the non-invasive lung adenocarcinomas and in a subset of the invasive adenocarcinomas.

With regard to immunophenotype, there were no clear differences between subepithelial and stromal myofibroblasts by $\alpha$-smooth muscle actin, desmin, h-caldesmon, and calponin stainings. Nevertheless, we could make the distinction between these two types of myofibroblasts by the pattern and location. The subepithelial myofibroblasts even with irregular proliferation were observed in a peculiar location beneath the cancer cells or encircling cancer cell nests, whereas the stromal myofibroblasts formed bundles between cancer cell nests.

It was interesting to note that the subepithelial myofibroblasts were lost in most of the invasive carcinomas, although some cases showed retention of these cells in the invasive area. We classified lung adenocarcinomas into three groups (groups I to III) according to the pattern of retention of the subepithelial myofibroblasts. This classification proved to be useful in defining a subset of invasive adenocarcinomas with excellent prognosis.

Studies have shown that lung adenocarcinomas with predominant bronchioloalveolar carcinoma area show good prognosis. ${ }^{21,22}$ However, histological criteria for distinguishing between non-invasive and invasive tumor remain somewhat ambiguous. Microscopic features, such as desmoplasia, disruption of elastic fibers, presence of papillary, or solid tumor structures have been considered as indicators of invasion of lung adenocarcinomas; ${ }^{8,23-25}$ however, there is no consensus yet among pathologists as to what should be deemed as the real evidence of invasion. Also, the nature of stromal changes may not be the same for all invasive adenocarcinomas. Thus, a more objective method will be required to assess the stromal changes that occur during cancer invasion. In this setting, immunohistochemistry may provide useful information in addition to elastic stains. Disruption and altered composition of basement membrane, as detected by laminin-1 and collagen type IV, have been described in cancer cell invasion. ${ }^{26-28}$ This poses a challenge, however, as staining for laminin-1 and collagen type IV requires extensive proteinase treatment, a procedure, which is rather difficult to standardize. In this context, the evaluation of subepithelial myofibroblast pattern with $\alpha$-smooth muscle actin staining may provide a relatively easy means to analyze stromal changes in invasive lung adenocarcinomas. Also, our three-group classification is reasonably reproducible; the concordance between two observers was $87 \%$, and when we repeated our evaluation on a separate occasion, the concordance rate was $88 \%$.

Pathologists commonly consider emergence of activated fibroblasts ${ }^{4}$ or desmoplasia ${ }^{23}$ as evidence of invasion. In this study, however, invasion was not 

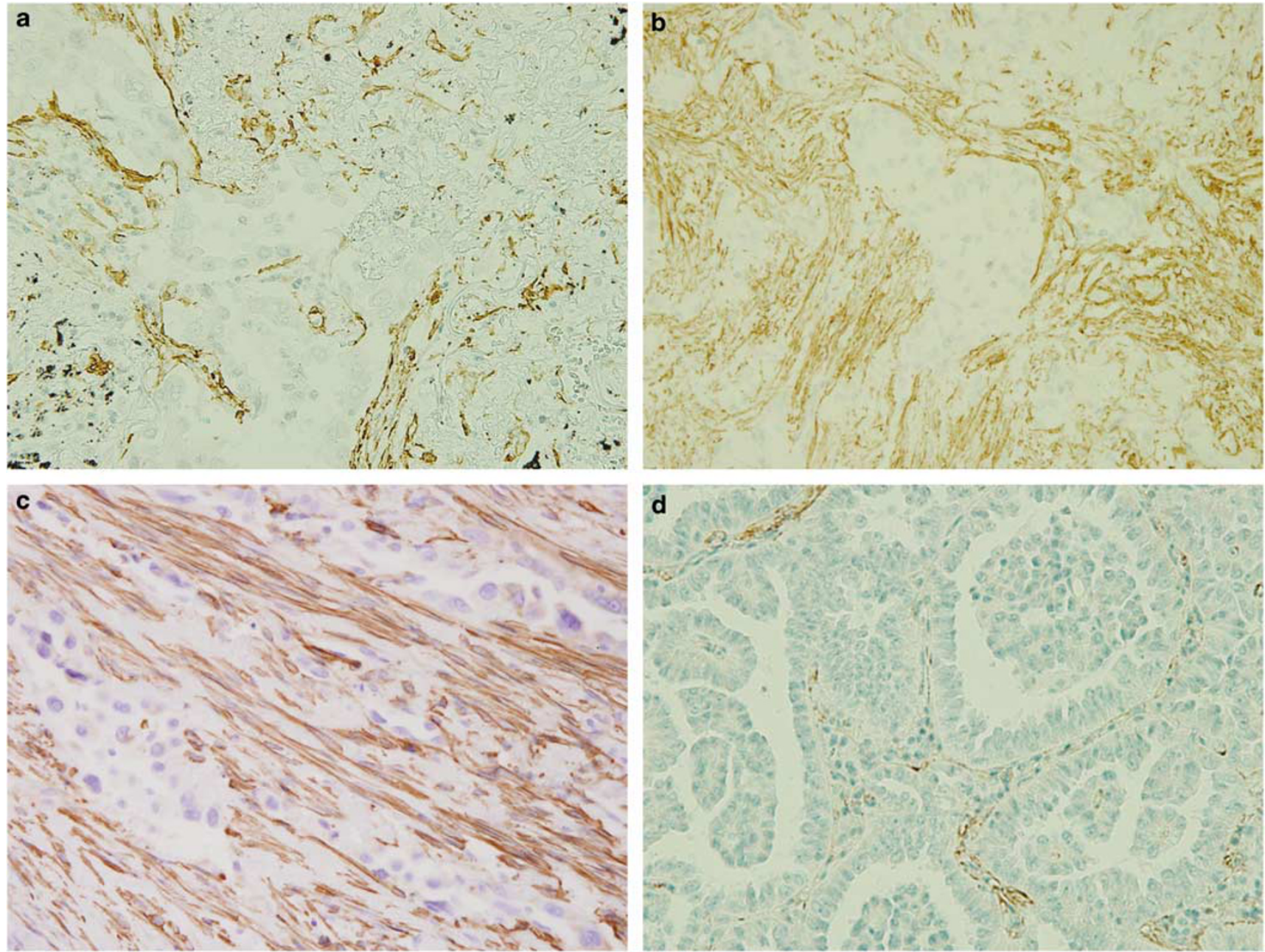

Figure 4 Subepithelial myofibroblasts in invasive lung adenocarcinomas ( $\alpha$-smooth muscle actin staining). In areas of central collapse and fibrosis, the myofibroblastic layer around the neoplastic acinar structure was discontinuous (a). Irregular proliferation obscuring the sharp contour of the myofibroblastic layer was also noted (b). In frankly invasive adenocarcinoma, subepithelial myofibroblasts were completely lost and bundles of stromal myofibroblasts emerged (c). In papillary adenocarcinomas, subepithelial myofibroblasts were only faintly visible in the preexisting alveolar structure (d). Subepithelial myofibroblasts were completely absent in the papillary structures (d).

Table 1 Histological features and subepithelial myofibroblast retention pattern

\begin{tabular}{lrrcr}
\hline Histological type & $\mathrm{n}$ & \multicolumn{3}{c}{$\begin{array}{c}\text { Subeipithelial myofibroblast } \\
\text { retention pattern }\end{array}$} \\
\cline { 3 - 5 } & & Group I & Group II & Group III \\
& & 13 & 0 & 0 \\
BAC & 13 & 11 & 8 & 1 \\
Mixed & 20 & 24 & 20 & 13 \\
Papillary & 57 & 1 & 1 & 2 \\
Acinar & 4 & 2 & 4 & 16 \\
Solid & 22 & & & \\
\hline
\end{tabular}

always accompanied by stromal myofibroblast proliferation. Some invasive cancers with central collapse manifested a loss of subepithelial myofibroblasts as the main stromal change, with no sign of myofibroblast proliferation in the stroma. We were also interested to note that some papillary adenocarcinomas exhibited a loss of subepithelial myofibroblasts as the prominent stromal change, with no stromal myofibroblast proliferation. In some papillary adenocarcinomas, the subepithelial myofibroblasts were lost even in the apparently intact preexisting alveolar structure. This tendency of a loss of subepithelial myofibroblasts without any induction of stromal myofibroblasts may be a peculiar feature in a subset of invasive adenocarcinomas.

Though data on subepithelial myofibroblasts in lung adenocarcinomas are lacking, there are detailed reports on subepithelial myofibroblastsoften referred to as 'pericryptal fibroblasts'in normal colonic mucosa and colorectal adenomas. ${ }^{29-32}$ In fact, the features of subepithelial myofibroblasts in lung adenocarcinoma are quite similar to those reported for pericryptal fibroblasts 
Table 2 Correlations between subepithelial myofibroblast retention pattern (group I to III) and clinicopathological factors in invasive adenocarcinoma

\begin{tabular}{|c|c|c|c|c|c|}
\hline \multirow[t]{2}{*}{ Clinical feature } & \multirow[t]{2}{*}{$\mathrm{n}$} & \multicolumn{3}{|c|}{$\begin{array}{l}\text { Subepithelial myofibroblast } \\
\text { pattern }\end{array}$} & \multirow[t]{2}{*}{$\mathrm{P}$-value } \\
\hline & & Group I & Group II & Group III & \\
\hline \multicolumn{6}{|l|}{ Pathological stage } \\
\hline I & 69 & 36 & 22 & 11 & $<0.0001$ \\
\hline $\mathrm{II}+\mathrm{III}+\mathrm{IV}$ & 34 & 2 & 11 & 21 & \\
\hline \multicolumn{6}{|l|}{ Nodal involvement } \\
\hline Negative (pNo) & 78 & 37 & 26 & 15 & $<0.0001$ \\
\hline Positive (pN1,2) & 25 & 1 & 7 & 17 & \\
\hline \multicolumn{6}{|l|}{ Pleural invasion } \\
\hline Negative & 53 & 28 & 17 & 8 & 0.000 \\
\hline Positive & 50 & 10 & 16 & 24 & \\
\hline \multicolumn{6}{|c|}{ Pulmonary metastasis } \\
\hline Negative & 90 & 35 & 28 & 27 & 0.54 \\
\hline Positive & 13 & 3 & 5 & 5 & \\
\hline \multicolumn{6}{|l|}{ Differentiation } \\
\hline Well & 59 & 34 & 20 & 5 & $<0.0001$ \\
\hline Moderate-poor & 44 & 4 & 13 & 27 & \\
\hline \multicolumn{6}{|l|}{ Vascular invasion } \\
\hline Negative & 69 & 34 & 23 & 12 & $<0.0001$ \\
\hline Positive & 34 & 4 & 10 & 20 & \\
\hline \multicolumn{6}{|l|}{ Lymphatic invasion } \\
\hline Negative & 73 & 38 & 23 & 12 & $<0.000$ \\
\hline Positive & 30 & 0 & 10 & 20 & \\
\hline
\end{tabular}

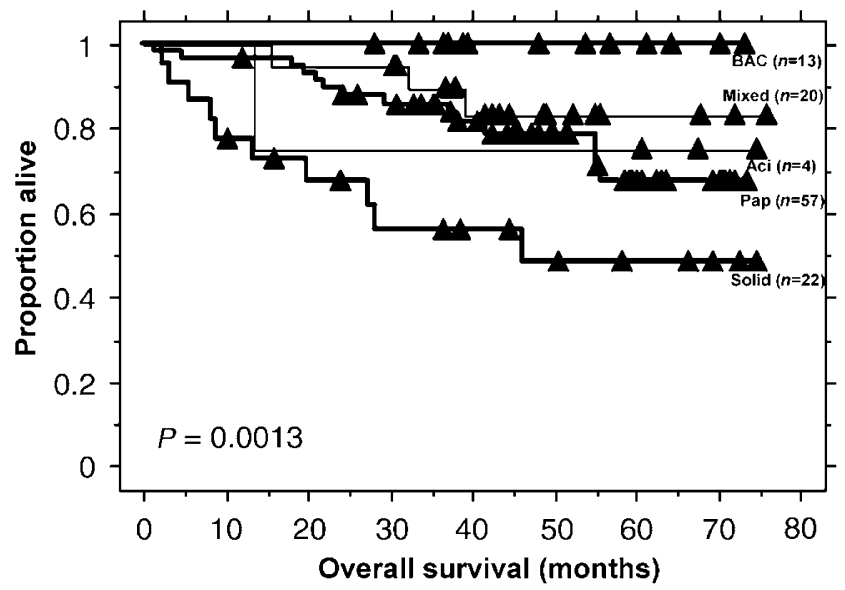

Figure 5 Patient survival for the whole study group according to the histological subtyping. BAC indicates bronchioloalveolar carcinoma; Mixed, mixed type adenocarcinoma; Aci, acinar adenocarcinoma; Pap, papillary adenocarcinoma; Solid, solid adenocarcinoma with mucin.

in colorectal epithelial tumors. Pericryptal fibroblasts are more abundant in adenoma than in normal colon, and gradually decrease during the progression from adenoma to intramucosal carcinoma to submucosal invasive carcinoma. ${ }^{29-32}$ In invasive carcinomas they are virtually absent. ${ }^{29}$ Pericryptal a

a Invasive adenocarcinoma cases (all stages)
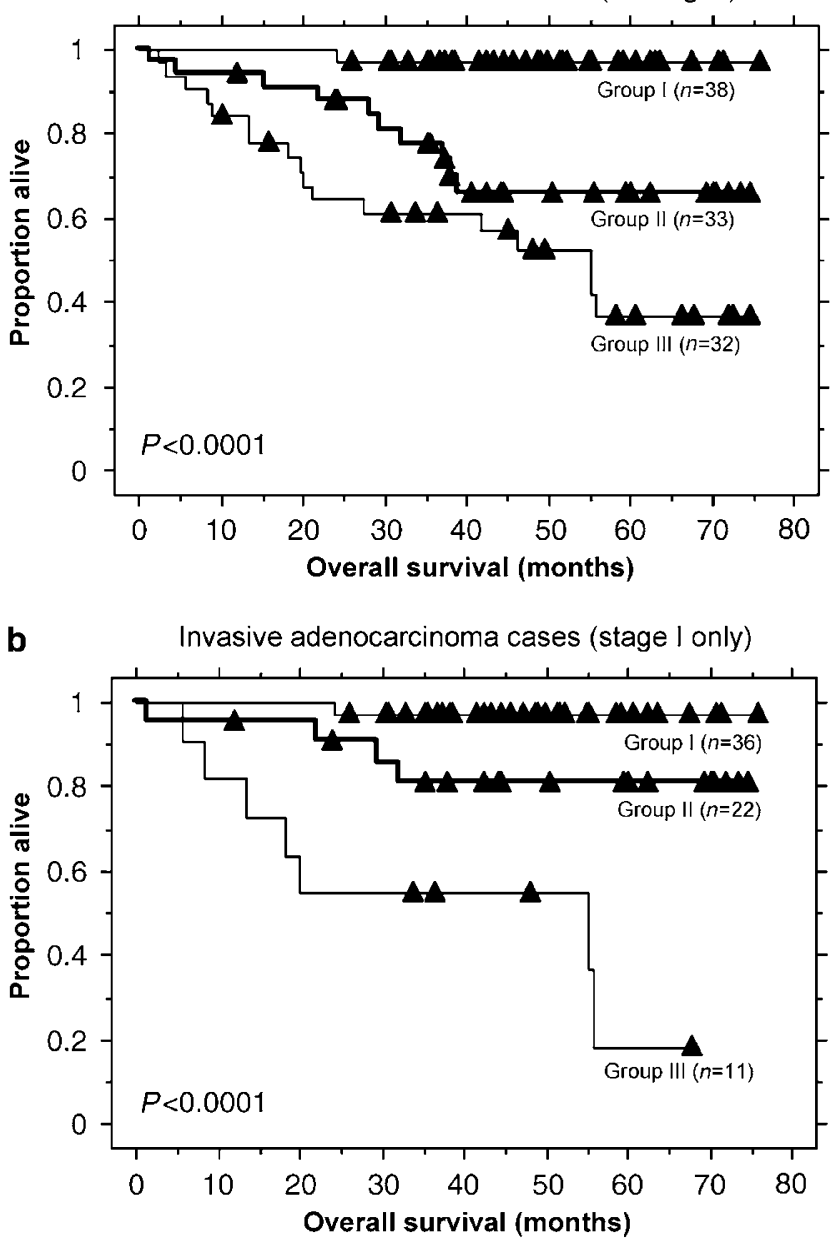

Figure 6 Patient survival according to the classification by the myofibroblastic pattern. (a) Survival curves for invasive adenocarcinomas (all stages). (b) Survival curves for invasive adenocarcinomas (stage I patient subgroup only).

fibroblasts are also absent in normal gastric mucosa. ${ }^{33}$ Interestingly, however, they appear in intestinal metaplastic glands, but disappear in the neoplastic glands of gastric adenocarcinomas of intestinal type. $^{33}$

Postoperative adjuvant chemotherapy, such as platinum-based chemotherapy, is now a standard therapy for completely resected non-small cell lung cancer, including early-stage cases. ${ }^{34,35}$ Postoperative adjuvant therapy, however, is not necessarily required for all stage IB cases. ${ }^{36}$ Patients with pure bronchioloalveolar carcinoma or minimally invasive cancer, for example, can be successfully treated with surgery alone. ${ }^{4,21,22}$ In this setting, analysis of subepithelial myofibroblasts by $\alpha$-smooth muscle actin staining might be helpful for identifying a subset of invasive adenocarcinomas requiring no postoperative chemotherapy, ie, tumors with myofibroblast pattern of group I. Conversely, stage I patients with tumors showing myofibroblastic pattern of group III may be treated with postoperative adjuvant therapy, as these patients have a much 
Table 3 (A) Univariate analysis and (B) multivariate analysis, stratified for stage

\begin{tabular}{lccc}
\hline Pathological factors & $\begin{array}{c}\text { Hazard } \\
\text { ratio }\end{array}$ & $\begin{array}{c}\text { 95\% confidence } \\
\text { interval }\end{array}$ & P-value \\
\hline (A) & & & \\
$\quad$ Group II+III vs group I & 9.90 & $2.342-41.67$ & $\underline{0.018}$ \\
$\quad$ Vascular invasion & 2.98 & $1.431-6.211$ & $\underline{0.0035}$ \\
$\quad$ Lymphatic invasion & 2.87 & $1.379-5.952$ & $\underline{0.0047}$ \\
$\quad$ Pleural invasion & 2.86 & $1.299-6.289$ & $\underline{0.0090}$ \\
$\quad$ Size & 1.37 & $1.162-1.611$ & $\underline{0.0002}$ \\
Differentiation & 1.28 & $0.613-2.653$ & 0.5145 \\
(Poor+mod vs well) & & & \\
$\quad$ Stage II+III vs stage I & 3.07 & $1.471-6.369$ & $\underline{0.0028}$ \\
$\quad$ & & & \\
(B) & & & \\
$\quad$ Group II+III vs group I & 6.07 & $1.305-28.18$ & $\underline{0.0214}$ \\
$\quad$ Pleural invasion & 1.36 & $0.559-3.301$ & 0.4994 \\
$\quad$ Vascular invasion & 1.32 & $0.578-3.011$ & 0.5105 \\
$\quad$ Size & 1.16 & $0.956-1.411$ & 0.1307 \\
$\quad$ Lymphatic invasion & 1.06 & $0.454-2.488$ & 0.8897 \\
\hline
\end{tabular}

The underlined values indicate those that are statistically significant.

poorer prognosis (5-year survival of $18 \%$ compared with 97 and $81 \%$ for group I and II, respectively).

In conclusion, we reported the presence of subepithelial myofibroblasts in pure bronchioloalveolar carcinoma and a subset of invasive lung adenocarcinomas. The retention of subepithelial myofibroblasts in invasive lung adenocarcinoma cases was associated with low rates of lymphatic, vascular, and pleural invasion, lymph node metastasis, and excellent prognosis. Analysis of subepithelial myofibroblast pattern by $\alpha$-smooth muscle actin staining may be useful for identifying invasive adenocarcinomas with excellent prognosis.

\section{Acknowledgements}

We thank Ms Yukie Ozawa for immunohistochemistry, Mr Tatsuo Sakurai and Ms Kiyomi Hidano for electron microscopy, and Ms Makiko Naka Mieno for advice on statistical analysis. This study was supported in part by the Foundation for Development of Community, the Vehicle Racing Commemorative Foundation, the Ministry of Health, Labor, and Welfare, and a grant-in-aid for scientific research from the Ministry of Education, Culture, Sports, Science, and Technology.

\section{References}

1 Statistics and Information Department. Vital Statistics, 2000. Ministry of Health, Labor and Welfare: Tokyo, 2001.

2 Janssen-Heijnen ML, Coebergh JW. Trends in incidence and prognosis of the histological subtypes of lung cancer in North America, Australia, New Zealand and Europe. Lung Cancer 2001;31:123-137.

3 Naruke T, Tsuchiya R, Kondo H, et al. Prognosis and survival after resection for bronchogenic carcinoma based on the 1997 TNM-staging classification: the Japanese experience. Ann Thorac Surg 2001;71: 1759-1764.

4 Noguchi M, Morikawa A, Kawasaki M, et al. Small adenocarcinoma of the lung. Histologic characteristics and prognosis. Cancer 1995;75:2844-2852.

5 Micke P, Ostman A. Tumour-stroma interaction: cancerassociated fibroblasts as novel targets in anti-cancer therapy? Lung Cancer 2004;45(Suppl 2):S163-S175.

6 De Wever O, Mareel M. Role of tissue stroma in cancer cell invasion. J Pathol 2003;200:429-447.

7 Yokota J, Kohno T. Molecular footprints of human lung cancer progression. Cancer Sci 2004;95:197-204.

8 Terasaki H, Niki T, Matsuno Y, et al. Lung adenocarcinoma with mixed bronchioloalveolar and invasive components: clinicopathological features, subclassification by extent of invasive foci, and immunohistochemical characterization. Am J Surg Pathol 2003;27: 937-951.

9 Sobin L, Wittekind L. TNM Classification of Malignant Tumors. John Wiley \& Sons: New York, 1997.

10 Travis WD, Brambilla E, Muller-Hermelink HK, et al. World Health Organization Classification of Tumors. Pathology and Genetics of Tumors of the Lung, Pleura, Thymus and Heart. International Agency for Research on Cancer Press: Lyon, 2004.

$11 \mathrm{Kim}$ YH, Ishii G, Goto K, et al. Dominant papillary subtype is a significant predictor of the response to gefitinib in adenocarcinoma of the lung. Clin Cancer Res 2004;10:7311-7317.

12 Takano T, Ohe Y, Sakamoto H, et al. Epidermal growth factor receptor gene mutations and increased copy numbers predict gefitinib sensitivity in patients with recurrent non-small-cell lung cancer. J Clin Oncol 2005;23:6829-6837.

13 Matsubara D, Niki T, Ishikawa S, et al. Differential expression of S100A2 and S100A4 in lung adenocarcinomas: clinicopathological significance, relationship to p53 and identification of their target genes. Cancer Sci 2005;96:844-857.

14 Nakamura Y, Niki T, Goto A, et al. c-Met activation in lung adenocarcinoma tissues: an immunohistochemical analysis. Cancer Sci 2007;98:1006-1013.

15 Suzuki K, Yokose T, Yoshida J, et al. Prognostic significance of the size of central fibrosis in peripheral adenocarcinoma of the lung. Ann Thorac Surg 2000; 69:893-897.

16 Kapanci Y, Assimacopoulos A, Irle C, et al. 'Contractile interstitial cells' in pulmonary alveolar septa: a possible regulator of ventilation-perfusion ratio? Ultrastructural, immunofluorescence, and in vitro studies. J Cell Biol 1974;60:375-392.

17 Noguchi A, Reddy R, Kursar JD, et al. Smooth muscle isoactin and elastin in fetal bovine lung. Exp Lung Res 1989;15:537-552.

18 Kapanci Y, Ribaux C, Chaponnier C, et al. Cytoskeletal features of alveolar myofibroblasts and pericytes in normal human and rat lung. J Histochem Cytochem 1992;40:1955-1963.

19 Sato M, Ozeki Y, Aida S, et al. Expression of alphasmooth muscle actin in small bronchioloalveolar adenocarcinoma of the lung: assessment and comparison with noguchi criteria. Oncol Rep 1999;6: 1217-1221.

20 Honda T, Ota H, Sano K, et al. Alveolar shrinkage in bronchioloalveolar carcinoma without central fibrosis. Lung Cancer 2002;36:283-288. 
21 Higashiyama M, Kodama K, Yokouchi H, et al. Prognostic value of bronchiolo-alveolar carcinoma component of small lung adenocarcinoma. Ann Thorac Surg 1999;68:2069-2673.

22 Sakao Y, Miyamoto H, Sakuraba M, et al. Prognostic significance of a histologic subtype in small adenocarcinoma of the lung: the impact of nonbronchioloalveolar carcinoma components. Ann Thorac Surg 2007;83:209-214.

23 Maeshima AM, Niki T, Maeshima A, et al. Modified scar grade: a prognostic indicator in small peripheral lung adenocarcinoma. Cancer 2002;95:2546-2554.

24 Eto T, Suzuki H, Honda A, et al. The changes of the stromal elastotic framework in the growth of peripheral lung adenocarcinomas. Cancer 1996;77:646-656.

25 Yokose T, Suzuki K, Nagai K, et al. Favorable and unfavorable morphological prognostic factors in peripheral adenocarcinoma of the lung $3 \mathrm{~cm}$ or less in diameter. Lung Cancer 2000;29:179-188.

26 Goto K, Yokose T, Kodama $\mathrm{T}$, et al. Detection of early invasion on the basis of basement membrane destruction in small adenocarcinomas of the lung and its clinical implications. Mod Pathol 2001;14: 1237-1245.

27 Kumaki F, Matsui K, Kawai T, et al. Expression of matrix metalloproteinases in invasive pulmonary adenocarcinoma with bronchioloalveolar component and atypical adenomatous hyperplasia. Am J Pathol 2001;159:2125-2135.

28 Nakano KY, Iyama KI, Mori T, et al. Loss of alveolar basement membrane type IV collagen alpha3, alpha4, and alpha5 chains in bronchioloalveolar carcinoma of the lung. J Pathol 2001;194:420-427.

29 Powell DW, Mifflin RC, Valentich JD, et al. Myofibroblasts. II. Intestinal subepithelial myofibroblasts. Am J Physiol 1999;277:C183-C201.

30 Yao T, Tsuneyoshi M. Significance of pericryptal fibroblasts in colorectal epithelial tumors: a special reference to the histologic features and growth patterns. Hum Pathol 1993;24:525-533.

$31 \mathrm{Li} \mathrm{A}$, Hasui $\mathrm{K}$, Yonezawa $\mathrm{S}$, et al. Immunohistochemical analysis of pericryptal fibroblast sheath and proliferating epithelial cells in human colorectal adenomas and carcinomas with adenoma components. Pathol Int 1999;49:426-434.

32 Sappino AP, Dietrich PY, Skalli O, et al. Colonic pericryptal fibroblasts. Differentiation pattern in embryogenesis and phenotypic modulation in epithelial proliferative lesions. Virchows Arch A Pathol Anat Histopathol 1989;415:551-557.

33 Mutoh H, Sakurai S, Satoh K, et al. Pericryptal fibroblast sheath in intestinal metaplasia and gastric carcinoma. Gut 2005;54:33-39.

34 Pisters KM. Adjuvant chemotherapy for non-small-cell lung cancer-the smoke clears. N Engl J Med 2005;352: 2640-2642.

35 Kato H, Ichinose Y, Ohta M, et al. A randomized trial of adjuvant chemotherapy with uracil-tegafur for adenocarcinoma of the lung. N Engl J Med 2004;350:1713-1721.

36 Jones DR, Daniel TM, Denlinger CE, et al. Stage IB nonsmall cell lung cancers: are they all the same? Ann Thorac Surg 2006;81:1958-1962. 